\title{
A Cardy-like formula for rotating black holes with planar horizon
}

\author{
Moisés Bravo Gaete, ${ }^{a}$ Luis Guajardo ${ }^{b}$ and Mokhtar Hassaïne ${ }^{b}$ \\ ${ }^{a}$ Facultad de Ciencias Básicas, Universidad Católica del Maule, \\ Casilla 617, Talca, Chile \\ ${ }^{b}$ Instituto de Matemática y Fisica, Universidad de Talca, \\ Casilla 747, Talca, Chile \\ E-mail: mbravo@ucm.cl, luis.guajardo.r@gmail.com, \\ hassaine@inst-mat.utalca.cl
}

ABSTRACT: We show that the semiclassical entropy of $D$-dimensional rotating (an)isotropic black holes with planar horizon can be successfully computed according to a Cardy-like formula. This formula does not refer to any central charges but instead involves the vacuum energy which is identified with a gravitational bulk soliton. The soliton is obtained from the non-rotating black hole solution by means of a double analytic continuation. The robustness of the Cardy-like formula is tested with numerous and varied examples, including AdS, Lifshitz and hyperscaling violation planar black holes.

Keywords: Black Holes, Classical Theories of Gravity

ARXIV EPRINT: 1702.02416 


\section{Contents}

1 Introduction 1

2 General formula for the asymptotic growth of the number of states 4

3 Corroborating the Cardy-like formula in the isotropic case, $z=1 \quad 6$

3.1 Stationary cylindrical black holes $\quad 7$

3.2 Black hole with a super-renormalizable self-interacting scalar field in 3D 8

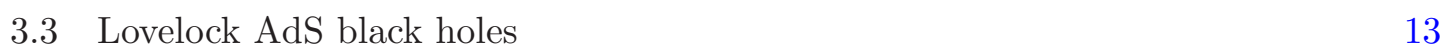

$\begin{array}{ll}3.4 \text { Hyperscaling violation black hole in } D \text { dimensions } & 15\end{array}$

3.5 Hyperscaling violation black hole with higher-order gravity theory 16

4 Testing the Cardy-like formula in the anisotropic case $\quad 17$

$\begin{array}{lll}4.1 & \text { Lifshitz black holes with higher-order gravity theories } & 17\end{array}$

$\begin{array}{ll}\text { 4.2 Charged anisotropic black holes with two Abelian gauge fields } & 19\end{array}$

$\begin{array}{ll}4.3 & \text { Turning on the angular momentum } 20\end{array}$

$\begin{array}{lll}5 & \text { Summary and concluding remarks } & 21\end{array}$

\section{Introduction}

Since the seminal works of Bekenstein and Hawking [1, 2], black holes are believed to behave as thermodynamic objects with characteristic temperature and entropy. A natural question has then emerged concerning the statistical interpretation of the black hole entropy. One of the first results that has shed some light on this problem was the observation that the asymptotic symmetries of the three-dimensional AdS space consist in two copies of the Virasoro algebra with a central charge [3]. This latter corresponds to the symmetry group of a two-dimensional CFT and, in this case, the Cardy formula is well-appropriate to express the asymptotic density of states [4]. An important manifestation of the AdS/CFT correspondence was then provided by showing that the Cardy formula applied for the BTZ black hole [5] correctly reproduces the expression of the Bekenstein-Hawking entropy [6]. Soon after, this approach was generalized for higher-dimensional black holes having a twodimensional CFT dual in the case of standard General Relativity [7] as well as in presence of higher-derivative corrections [8].

Extensions of the Cardy formula have been considered and studied in the current literature. Among other, one can mention the higher-dimensional generalization of the Cardy formula which applied for strongly coupled field theories having an AdS dual [9]. There also exist extensions of the Cardy formula with applications for three-dimensional spacetimes that are not AdS like the warped AdS spaces [10] or anisotropic spacetimes, 
namely the Lifshitz spacetimes [11] or the hyperscaling violation geometries [12-14]. The interest on anisotropic spacetimes has considerably grown up this last decade essentially due to the will of extending the ideas underlying the gauge/gravity duality to strongly coupled field theories with an anisotropic scaling symmetry [15]. Notice also that the cases of three-dimensional black holes have permitted a better comprehension of the holographic derivation of the entropy by highlighting the prominent role played by the soliton, see refs. [16-18]. This observation is not in contradiction with the standard derivation of the Cardy formula for which the ground state is implicitly assumed to be the three-dimensional AdS spacetime. Nevertheless, this assumption is in general valid only for the vacuum sector but not in the hairy sector which possesses a different ground state. It is then more judicious to deal with a Cardy formula written in terms of the vacuum energy rather than the central charges. Unfortunately, it is not possible a priori to find out the vacuum energy of the putative field theory. However, as stressed in refs. [16-18], the vacuum energy can be identified with the mass of a bulk soliton constructed from the black hole through a double Wick rotation in the same way that the AdS soliton [19], which reinforces the importance of the role played by the soliton. Importance also confirmed in the Lifshitz case [11] where the robustness of the Lifshitz Cardy formula has been tested successfully for three-dimensional Lifshitz black holes with a nonminimally scalar field [20].

One of the aim of this paper is precisely to confirm the importance of the gravitational soliton. We will highlight this importance in the case of rotating black holes in arbitrary dimension $D$ with a planar base manifold. This restriction on the horizon's topology is justified by the fact that the soliton can be easily constructed from the black hole by a double analytic continuation similar to the one operated in the AdS soliton [19]. With the view of achieving this task, we will be interested on higher-dimensional extensions of the Cardy formula for field theories satisfying the following two assumptions: (i) the field theory possibly displays a hyperscaling violation reflected by the fact that the thermal entropy $\mathcal{S}$ scales with respect to the temperature as $\mathcal{S} \sim T^{\frac{d_{\text {eff }}}{z}}$, and (ii) the ground state for the field theory is identified with a bulk soliton which is regular everywhere and devoid of any integration constant. Here, $d_{\mathrm{eff}}$ is an effective spatial dimensionality for the dual theory (related to the dimension of the stress-energy tensor) which measures the possible deviation from the spatial dimension and $z$ is the Lifshitz dynamical exponent. In the standard AdS situation, the "effective" spatial dimension $d_{\mathrm{eff}}=D-2$ and the dynamical exponent $z=1$. Under these hypothesis, formulas for the asymptotic growth of the number of states have been obtained in the non-rotating case in [12-14], and their spinning generalizations in the isotropic case $z=1$ were found in [21]. In the present work, we extend this formula for a generic dynamical exponent $z$. The resulting Cardy-like formula makes no mention to any central charge but instead involves the mass of the ground state which is identified with the nonrotating gravitational soliton. Nevertheless, since a very little is known about the putative field theories, we propose to corroborate the validity of the Cardy-like formula considering gravity theories whose spectrum of solutions contain black holes whose entropy exhibits a power law temperature as described in the hypothesis (i) as well as regular solitons (ii). This inspection will be done for different classes of black hole solutions with different asymptotic behaviors (rotating AdS, Lifshitz and hyperscaling violation black 
holes with a planar base manifold) by comparing the gravitational entropy with the entropy field expression involving the effective spatial dimension and the vacuum energy. In all our examples, the spinning planar black holes are derived from static configurations through a Lorentz boost and the vacuum energy corresponds to the mass of the gravitational soliton obtained from the nonrotating black hole through a double Wick rotation. We will also extend these results to the case of charged planar black holes, where again the ground state is identified with the soliton derived from the neutral and nonrotating black hole. Since the soliton is devoid of any integration constant, its mass will be computed using the quasilocal generalization of the ADT formalism [22-26] as presented in refs. [27, 28]. One of the main result of these two last papers lies in the prescription of the off-shell ADT potential $\mathcal{Q}_{\mathrm{ADT}}^{\mu \nu}$ in terms of the off-shell Noether potential $K^{\mu \nu}$ and the surface term $\Theta^{\mu}$ arising from the variation of the action

$$
\sqrt{-g} \mathcal{Q}_{\mathrm{ADT}}^{\mu \nu}=\frac{1}{2} \delta K^{\mu \nu}-\xi^{[\mu} \Theta^{\nu]},
$$

where $\xi^{\mu}$ denotes the Killing vector. The corresponding conserved charge is computed to be

$$
Q(\xi)=\int d^{D-2} x_{\mu \nu}\left(\Delta K^{\mu \nu}(\xi)-2 \xi^{[\mu} \int_{0}^{1} d s \Theta^{\nu]}(\xi \mid s)\right),
$$

where $\Delta K^{\mu \nu}(\xi) \equiv K_{s=1}^{\mu \nu}(\xi)-K_{s=0}^{\mu \nu}(\xi)$ denotes the difference of the Noether potential between the black hole and the zero-mass solution, and $d^{D-2} x_{\mu \nu}$ represents the integration over the co-dimension two boundary. For the examples treated in this paper, the action can schematically be written as

$$
S=\int d^{D} x \sqrt{-g} \mathcal{L}\left(g, \phi, A_{(i)}\right),
$$

where $\phi$ is a scalar field (possibly a dilatonic field) with its usual kinetic term and $A_{(i)}=$ $A_{(i) \mu} d x^{\mu}$ stand for Abelian gauge fields or Proca fields. In this generic case, the boundary term and Noether potential needed to compute the charge (1.2) are given by

$$
\begin{aligned}
\Theta^{\mu} & =2 \sqrt{-g}\left[P^{\mu(\alpha \beta) \gamma} \nabla_{\gamma} \delta g_{\alpha \beta}-\delta g_{\alpha \beta} \nabla_{\gamma} P^{\mu(\alpha \beta) \gamma}+\frac{1}{2} \sum_{i}\left(\frac{\partial \mathcal{L}}{\partial\left(\partial_{\mu} A_{(i) \nu}\right)} \delta A_{(i) \nu}\right)+\frac{1}{2} \frac{\partial \mathcal{L}}{\partial\left(\partial_{\mu} \phi\right)} \delta \phi\right], \\
K^{\mu \nu} & =\sqrt{-g}\left[2 P^{\mu \nu \rho \sigma} \nabla_{\rho} \xi_{\sigma}-4 \xi_{\sigma} \nabla_{\rho} P^{\mu \nu \rho \sigma}-\sum_{i} \frac{\partial \mathcal{L}}{\partial\left(\partial_{\mu} A_{(i) \nu}\right)} \xi^{\sigma} A_{(i) \sigma}\right],
\end{aligned}
$$

where $P^{\mu \nu \rho \sigma}=\frac{\partial \mathcal{L}}{\partial R_{\mu \nu \rho \sigma}}$, and $R_{\mu \nu \rho \sigma}$ is the Riemann tensor.

The plan of the paper is organized as follows. In the next section, a general formula for the asymptotic growth of the number of states including the angular momentum is proposed. This generic Cardy-like formula involves the effective spatial dimension $d_{\text {eff }}$, the Lifshitz dynamical exponent $z$, the mass and angular momentum of the black hole as well as the vacuum energy which corresponds to the mass of the bulk soliton. In section 3, we corroborate the validity of the Cardy-like formula in the isotropic case $z=1$ with stationary cylindrical black holes. The case of a three-dimensional black hole solution of the Einstein equations with a source given by a self-interacting scalar field with a super-renormalizable potential is also treated in full details. Lovelock AdS black holes will also be inspected in 
order to reinforce the validity of the Cardy-like formula. To end the section 3 , two examples of hyperscaling violating black holes with different effective spatial dimensionality will be studied. In section 4 , we will deal with the anisotropic case $z \neq 1$. Lifshitz black holes solutions of higher-order gravity theories will be our first testing example while the case of charged anisotropic black holes produced by various dilaton fields will constitute our second class of example. Finally, the last section is devoted to the summary and to the concluding remarks. For simplicity, we have decided to fix the radius of curvature to unit, $l=1$, while the Newton gravitational constant $G$ is defined through the change $2 \kappa=16 \pi G$.

\section{General formula for the asymptotic growth of the number of states}

As recalled in the introduction, the asymptotic symmetries of $\mathrm{AdS}_{3}$ are represented by two copies of the Virasoro algebra with equal left and right moving central charges

$$
c^{+}=c^{-}=c=\frac{3 l}{2 G}=\frac{12 \pi}{\kappa},
$$

(in our convention $l=1$ and $2 \kappa=16 \pi G$ ), and the standard Cardy formula takes the following form

$$
S=2 \pi \sqrt{\frac{c}{6} \widetilde{\Delta}^{+}}+2 \pi \sqrt{\frac{c}{6} \widetilde{\Delta}^{-}},
$$

where $\widetilde{\Delta}^{ \pm}=\frac{1}{2}(M \pm J)$ are the eigenvalues of the left and right Virasoro operators. In this representation of the Cardy formula, it is implicitly assumed that the ground state is identified with the AdS spacetime. Nevertheless, the AdS spacetime is only a suitable ground state in the case of standard General Relativity, and this assumption is not longer valid in presence of source for example. Hence, it is more reasonable to deal with a Cardy formula involving the vacuum charge than the central charge. In the vacuum sector for standard General Relativity, the ground state is nothing but the three-dimensional AdS soliton whose mass is computed below (3.9) and gives $M_{\text {sol }}=-\pi / \kappa$. Finally, the standard Cardy formula (2.1) can be as well expressed as

$$
S=4 \pi \sqrt{-\frac{1}{2} M_{\mathrm{sol}}} \sqrt{\widetilde{\Delta}^{-}}+4 \pi \sqrt{-\frac{1}{2} M_{\mathrm{sol}}} \sqrt{\widetilde{\Delta}^{+}} .
$$

Notice that Cardy-like formulas involving the vacuum energy instead of the central charges have been proved to be very useful for examples where the ground state is not the threedimensional AdS spacetime, see e.g. [11-14, 16-18]. The matching between the gravitational entropy and the Cardy formula (2.2) is perfectly consolidated for three-dimensional black holes that are asymptotically AdS (even in the weaker sense). Nevertheless, as mentioned in the introduction, we are interested on generalizations of the Cardy formula that apply for field theories displaying an hyperscaling violation behavior such that the thermal entropy $\mathcal{S}$ scales w.r.t. the temperature $T$ as

$$
\mathcal{S} \sim T^{\frac{d_{\mathrm{eff}}}{z}}
$$


where $d_{\text {eff }}$ is an effective spatial dimensionality and $z$ is the Lifshitz exponent. In order to achieve this task, we closely follow the derivations done in refs. [12, 21]. The partition function $\mathcal{Z}$ defined on the torus of modulus $\tau$ such that $2 \pi \tau=2 \pi r e^{i \phi}$ and $2 \pi \bar{\tau}=2 \pi r e^{-i \phi}$ can be written as

$$
\mathcal{Z}[\tau, \bar{\tau}]=\operatorname{Tr}\left[e^{2 \pi i \tau L_{0}} e^{-2 \pi i \bar{\tau} \bar{L}_{0}}\right],
$$

with $L_{0}+\bar{L}_{0}=M$ and $L_{0}-\bar{L}_{0}=J$. The density of states $\rho(M, J)$ can be obtained by taking an inverse Laplace transform yielding

$$
\rho(M, J)=\int d r d \phi \mathcal{Z}[r, \phi] \exp \left[-2 \pi i r e^{i \phi} L_{0}+2 \pi i r e^{-i \phi} \bar{L}_{0}\right]
$$

In the microcanonical ensemble, the entropy is basically the logarithm of the density of states $\mathcal{S} \sim \log \rho(M, J)$. Defining the quantity

$$
\mathcal{Z}_{0}[r, \phi]=\operatorname{Tr}\left\{\exp \left[2 \pi i r e^{i \phi}\left(L_{0}-\frac{M_{\text {sol }}}{2}\right)-2 \pi i r e^{-i \phi}\left(\bar{L}_{0}-\frac{M_{\text {sol }}}{2}\right)\right]\right\}
$$

and assuming that $\mathcal{Z}_{0}$ presents the following modular invariance

$$
\mathcal{Z}_{0}\left[-\frac{1}{r^{\frac{d_{\mathrm{eff}}}{z}}},-\phi\right]=\mathcal{Z}_{0}[r, \phi]
$$

the density of states $\rho(M, J)$ can be re-written as

$\rho(M, J)=\int d r d \phi \mathcal{Z}_{0}\left[-\frac{1}{r^{\frac{d_{\mathrm{eff}}}{z}}},-\phi\right] \exp \left[-\frac{\pi i M_{\mathrm{sol}}}{r^{\frac{d_{\mathrm{eff}}}{z}}} e^{-i \phi}+\frac{\pi i M_{\mathrm{sol}}}{r^{\frac{d_{\mathrm{eff}}}{z}}} e^{i \phi}-2 \pi i r e^{i \phi} L_{0}+2 \pi i r e^{-i \phi} \bar{L}_{0}\right]$.

Now, as usual, this last expression can be evaluated using a saddle-point approximations for $r$ and $\phi$, and assuming that $\mathcal{Z}_{0}$ varies slowly, one gets

$$
\begin{aligned}
S= & \pi \sqrt{\frac{d_{\mathrm{eff}}+z}{z}}\left[\left(-2 M_{\mathrm{sol}}\right)^{z} \frac{1}{d_{\mathrm{eff}}^{d_{\mathrm{eff}}}}\right]^{\frac{1}{z+d_{\mathrm{eff}}}}\left(\sqrt{\left(d_{\mathrm{eff}}+z\right)^{2} M^{2}-4 d_{\mathrm{eff}} z J^{2}}+\left(d_{\mathrm{eff}}+z\right) M\right)^{\frac{1}{2}} \\
& \times\left(\sqrt{\left(d_{\mathrm{eff}}+z\right)^{2} M^{2}-4 d_{\mathrm{eff}} z J^{2}}-\left(d_{\mathrm{eff}}-z\right) M\right)^{\frac{d_{\mathrm{eff}}-z}{2\left(d_{\mathrm{eff}}+z\right)}}
\end{aligned}
$$

This formula constitutes the extension of the Cardy formula (2.2) in arbitrary dimension for a field theory with a spatial effective dimension $d_{\text {eff }}$ and dynamical exponent $z$.

Let us see the consistency of this expression with known formulas. First of all, in the isotropic case $z=1$, the expression (2.4) is compatible with the Cardy formula (2.2) in the standard AdS case in three dimensions (which corresponds to $d_{\mathrm{eff}}=1$ ) as well as with the formula derived in [21] for hyperscaling violation metric. On the other hand, in the non-rotating case with anisotropy, i.e. $J=0$ with $z \neq 1$, the formula (2.4) reproduces the Lifshitz Cardy formula in three dimensions with $d_{\text {eff }}=1$, see [11], and also the generic formula for hyperscaling violation metric [12-14]. 
In the electrically charged case, the Cardy-like formula (2.4) becomes

$$
\begin{aligned}
S= & \pi \sqrt{\frac{d_{\mathrm{eff}}+z}{z}\left[\left(-2 M_{\mathrm{sol}}\right)^{z} \frac{1}{d_{\mathrm{eff}}^{d_{\mathrm{eff}}}}\right]^{\frac{1}{z+d_{\mathrm{eff}}}}} \\
& \times\left(\sqrt{\left(d_{\mathrm{eff}}+z\right)^{2}\left(M-\frac{1}{2} \phi_{e} Q_{e}\right)^{2}-4 d_{\mathrm{eff}} z J^{2}}+\left(d_{\mathrm{eff}}+z\right)\left(M-\frac{1}{2} \phi_{e} Q_{e}\right)\right)^{\frac{1}{2}} \\
& \times\left(\sqrt{\left(d_{\mathrm{eff}}+z\right)^{2}\left(M-\frac{1}{2} \phi_{e} Q_{e}\right)^{2}-4 d_{\mathrm{eff}} z J^{2}}-\left(d_{\mathrm{eff}}-z\right)\left(M-\frac{1}{2} \phi_{e} Q_{e}\right)\right)^{\frac{d_{\mathrm{eff}-z}}{2\left(d_{\mathrm{eff}}+z\right)}}
\end{aligned}
$$

where $\phi_{e}$ denotes the electric potential while $Q_{e}$ stands for the electric charge.

\section{Corroborating the Cardy-like formula in the isotropic case, $z=1$}

In this section, we will be mainly concerned with planar black holes that are asymptotically AdS or exhibiting an hyperscaling violation behavior. In these cases, the asymptotic form of the metric can be parameterized as follows

$$
d s^{2}=\frac{1}{r^{\frac{2 \theta}{D-2}}}\left[-r^{2} d t^{2}+\frac{d r^{2}}{r^{2}}+r^{2} \sum_{i=1}^{D-2} d x_{i}^{2}\right],
$$

where $\theta$ represents the parameter responsible of the violation of the hyperscaling property, and $\theta=0$ will correspond to the planar AdS case. Note that, for the asymptotic metric (3.1), the isotropic transformations $t \rightarrow \lambda t, r \rightarrow \lambda^{-1} r$ and $x_{i} \rightarrow \lambda x_{i}$ are identified as an isometry in the AdS case, and as a conformal transformation for non vanishing $\theta$.

For this class of black holes, the dynamical exponent appearing in the Cardy-like formula (2.4) corresponds to the isotropic situation $z=1$ while the effective spatial dimension $d_{\mathrm{eff}}$ is given by $d_{\mathrm{eff}}=D-2$ in the AdS case $(\theta=0)$ otherwise it will depend explicitly on the violating parameter $\theta$. In the hyperscaling case, one of the difficulty is to correctly identify the functional dependence of the effective spatial dimension. For example, for Einstein gravity with scalar field source, the effective spatial dimension is $d_{\mathrm{eff}}=D-2-\theta$, while for higher-order gravity theories, this dependence may be different as shown below, see also $[13,14]$.

In what follows, we will treat various isotropic examples with the aim of testing the Cardy-like formula, starting from AdS planar black holes. As a first example, we examine $D$-dimensional stationary cylindrical black holes solutions of Einstein gravity with a negative cosmological constant. We also look at the case of a three-dimensional solution of Einstein gravity with a self-interacting scalar field with a super-renormalizable potential. The case of higher theories is also inspected through the analysis of Lovelock AdS black holes. In the second part, two examples of hyperscaling violation black holes will be studied. The first testing example is described by the Einstein gravity with a scalar field source corresponding to a spatial dimensionality $d_{\text {eff }}=D-2-\theta$. Next, in order to test the force of the Cardy-like formula with a different spatial dimensionality, we deal with an hyperscaling violating black hole solution of a pure quadratic gravity theory. 


\subsection{Stationary cylindrical black holes}

We start the corroborating study of the Cardy-like formula (2.4) with the case of the Einstein field equations in the presence of a negative cosmological constant

$$
G_{\mu \nu}-\frac{(D-1)(D-2)}{2} g_{\mu \nu}=0,
$$

and whose corresponding action is

$$
S[g]=\frac{1}{2 \kappa} \int d^{D} x \sqrt{-g}(R+(D-1)(D-2)) .
$$

We consider the higher-dimensional extension of the stationary cylindrical black hole found by Lemos [29] in four dimensions and reported in [30],

$$
\begin{aligned}
d s^{2}= & -F(r)\left(\Xi d t-\sum_{i=1}^{n} a_{i} d \phi_{i}\right)^{2}+r^{2} \sum_{i=1}^{n}\left(a_{i} d t-\Xi d \phi_{i}\right)^{2}+\frac{d r^{2}}{F(r)} \\
& -r^{2} \sum_{i<j}^{n}\left(a_{i} d \phi_{j}-a_{j} d \phi_{i}\right)^{2}+r^{2} \sum_{i=1}^{D-2-n} d x_{i}^{2} .
\end{aligned}
$$

Here $n=[(D-1) / 2]$ corresponds to the number of rotation parameters $a_{i}, \Xi=\sqrt{1+\sum_{i}^{n} a_{i}^{2}}$, and the metric function reads

$$
F(r)=r^{2}\left(1-\left(\frac{r_{h}}{r}\right)^{D-1}\right) .
$$

In four dimensions, the number of rotations is $n=1$, and the solution reduces to the stationary cylindrical black hole solution of Lemos [29].

As calculated in ref. [30], the entropy of the solution is

$$
\mathcal{S}=\frac{2 \pi \operatorname{Vol}\left(\Sigma_{D-2}\right) \Xi r_{h}^{D-2}}{\kappa},
$$

with mass and angular momenta given by

$$
M=\frac{\operatorname{Vol}\left(\Sigma_{D-2}\right)}{2 \kappa}\left((D-1) \Xi^{2}-1\right) r_{h}^{D-1}, \quad J_{i}=\frac{(D-1) \operatorname{Vol}\left(\Sigma_{D-2}\right)}{2 \kappa} \Xi a_{i} r_{h}^{D-1},
$$

where $\operatorname{Vol}\left(\Sigma_{D-2}\right)$ corresponds to the volume element of the $(D-2)$-dimensional Euclidean space.

In order to test the validity of the Cardy-like formula (2.4), one needs to construct the static soliton and to compute its mass through the quasilocal expression (1.2). As explained in the introduction, operating a double Wick rotation on the static version of the solution, that is eq. (3.3) with $a_{i}=0$, one gets the AdS soliton [19]

$$
d s^{2}=-r^{2} d t^{2}+\frac{d r^{2}}{f(r)}+f(r) d \varphi^{2}+r^{2} \sum_{i=1}^{D-3} d x_{i}^{2},
$$


with

$$
f(r)=r^{2}\left[1-\left(\frac{2}{(D-1) r}\right)^{D-1}\right] .
$$

The next step is to determine the mass of the soliton through the quasilocal formula (1.2) where the Killing vector field is $\xi^{t}=\partial_{t}$. The variation of the Noether potential and the surface term are given by

$$
\int_{0}^{1} d s \Theta^{r}=\frac{1}{2 \kappa}\left(\frac{2}{D-1}\right)^{D-1}, \quad \Delta K^{r t}\left(\xi^{t}\right)=-\frac{1}{\kappa}\left(\frac{2}{D-1}\right)^{D-1} .
$$

Finally, the mass of the $D$-dimensional AdS gravitational soliton reads

$$
M_{\mathrm{sol}}=-\frac{\operatorname{Vol}\left(\Sigma_{D-2}\right)}{2 \kappa}\left(\frac{2}{D-1}\right)^{D-1}
$$

and it is simple to check that the formula (2.4) where $J^{2}$ is now understood as $J^{2}=\sum_{i=1}^{n} J_{i}^{2}$ and where $d_{\mathrm{eff}}=D-2$ and $z=1$ correctly reproduces the gravitational entropy (3.4).

\subsection{Black hole with a super-renormalizable self-interacting scalar field in 3D}

We pursue our survey considering now a three-dimensional toy model whose action is described by the Einstein-Hilbert piece with a cosmological constant together with a nonminimally self-interacting scalar field

$$
S[g, \phi]=\int d^{3} x \sqrt{-g}\left(\frac{R-2 \Lambda}{2 \kappa}-\frac{1}{2} \nabla_{\mu} \phi \nabla^{\mu} \phi-\frac{1}{16} R \phi^{2}-U(\phi)\right) .
$$

The nonminimal coupling corresponds to the conformal one in three dimensions, and as it is well known the potential term which is compatible with the conformal invariance of the matter source is $U(\phi) \propto \phi^{6}$. Nevertheless, in our case, we chose a potential term breaking the conformal invariance of the matter action, and defined by all the powers lower than the conformal one (super-renormalizable potential), that is

$$
U(\phi)=\lambda_{1} \phi+\lambda_{2} \phi^{2}+\lambda_{3} \phi^{3}+\lambda_{4} \phi^{4}+\lambda_{5} \phi^{5}+\lambda_{6} \phi^{6} .
$$

The field equations obtained from the variation of the action (3.10) with respect to the metric and the scalar field are

$$
\begin{aligned}
G_{\mu \nu}+\Lambda g_{\mu \nu} & =\kappa\left(\nabla_{\mu} \phi \nabla_{\nu} \phi-g_{\mu \nu}\left(\frac{1}{2} \nabla_{\sigma} \phi \nabla^{\sigma} \phi+U\right)+\frac{1}{8}\left(g_{\mu \nu} \square-\nabla_{\mu} \nabla_{\nu}+G_{\mu \nu}\right) \phi^{2}\right), \\
\square \phi-\frac{1}{8} R \phi & =\frac{d U}{d \phi} .
\end{aligned}
$$

In ref. [32], the authors have derived a static black hole solution of the model described by (3.10)-(3.11)-(3.12) using a conformal machinery where the coupling constants are 
parameterized as follows

$$
\begin{aligned}
& \lambda_{1}=\frac{\left[(\mu-3)^{2}(4 \mu-3) \lambda^{4}+27(\mu-1)^{2}\right] \lambda \sqrt{2}}{18 \sqrt{\kappa}(\mu-1)^{2}\left(1-\lambda^{2}\right)^{5}} \\
& \lambda_{2}=-\frac{5 \lambda^{2}\left[\lambda^{2}(4 \mu-3)(\mu-3)^{2}+27(\mu-1)^{2}\right]}{72(\mu-1)^{2}\left(1-\lambda^{2}\right)^{5}} \\
& \lambda_{3}=\frac{5 \sqrt{2 \kappa} \mu^{3} \lambda^{3}}{54(\mu-1)^{2}\left(1-\lambda^{2}\right)^{5}}, \quad \lambda_{4}=-\frac{5 \kappa \lambda^{2}\left[(4 \mu-3)(\mu-3)^{2}+27(\mu-1)^{2} \lambda^{2}\right]}{576(\mu-1)^{2}\left(1-\lambda^{2}\right)^{5}} \\
& \lambda_{5}=\frac{\left[27(\mu-1)^{2} \lambda^{4}+(4 \mu-3)(\mu-3)^{2}\right] \sqrt{2} \lambda \kappa^{3 / 2}}{1152(\mu-1)^{2}\left(1-\lambda^{2}\right)^{5}}, \\
& \lambda_{6}=-\frac{\kappa^{2}\left[27(\mu-1)^{2} \lambda^{6}+(4 \mu-3)(\mu-3)^{2}\right]}{13824(\mu-1)^{2}\left(1-\lambda^{2}\right)^{5}}, \\
& \Lambda=-\frac{\left[27(\mu-1)^{2}+\lambda^{6}(4 \mu-3)(\mu-3)^{2}\right]}{27(\mu-1)^{2}\left(1-\lambda^{2}\right)^{5}}
\end{aligned}
$$

More precisely, as shown in [32], the action defined by (3.10)-(3.11)-(3.13) can be obtained from the conformally invariant action ${ }^{1}$ denoted by $\tilde{S}[\tilde{g}, \tilde{\phi}]$ and corresponding to the action (3.10) with the potential $U \propto \tilde{\phi}^{6}$ through a map parameterized by the factor $\lambda$, and both actions are related as follows

$$
S[g, \phi]=\left(1-\lambda^{2}\right) \tilde{S}[\tilde{g}, \tilde{\phi}] .
$$

In fact, the static solution reported in [32] was constructed using the one-parameter mapping with a seed configuration given by the solution of the conformally self-interacting version of the Martinez-Zanelli solution [33] found in [34]. Instead of writing down the static solution [32], we report its spinning extension obtained from the static configuration as usual in three dimensions through a Lorentz boost defined by

$$
t \rightarrow \frac{1}{\sqrt{1-\omega^{2}}}(t+\omega \varphi), \quad \varphi \rightarrow \frac{1}{\sqrt{1-\omega^{2}}}(\varphi+\omega t),
$$

and well-defined for $\omega^{2}<1$. The line element of the resulting rotating solution is given by

$$
d s^{2}=H^{2}(r)\left\{-N^{2}(r) F(r) d t^{2}+\frac{d r^{2}}{F(r)}+R^{2}(r)\left(d \varphi+N^{\varphi}(r) d t\right)^{2}\right\},
$$

where the metric functions and the scalar field read

$$
\begin{aligned}
N^{2}(r) & =r^{2} \frac{\left(1-\omega^{2}\right)}{\left(r^{2}-\omega^{2} F(r)\right)}, \quad R^{2}(r)=\frac{1}{\left(1-\omega^{2}\right)}\left(r^{2}-\omega^{2} F(r)\right), \quad N^{\varphi}(r)=\frac{\omega\left(r^{2}-F(r)\right)}{\left(r^{2}-\omega^{2} F(r)\right)}, \\
\phi(r) & =\sqrt{\frac{2}{\kappa H(r)}}\left[\sqrt{\frac{12(\mu-1) r_{h}}{\left(3(\mu-1) r_{h}-2 r \mu\right)}}+2 \lambda\right], \\
F(r) & =r^{2}\left[1+(\mu-1)\left(\frac{r_{h}}{r}\right)^{3}-\mu\left(\frac{r_{h}}{r}\right)^{2}\right], \quad H(r)=\left[\lambda \sqrt{\frac{3(\mu-1) r_{h}}{\left(3(\mu-1) r_{h}-2 r \mu\right)}}+1\right]^{2} .
\end{aligned}
$$

${ }^{1}$ There is a slight abuse of language in the sense that "by conformally invariant action", we mean that only the matter source involving the scalar field is invariant under the conformal transformations and not the gravity action. 
We now analyze the thermodynamical properties of the spinning solution through the Euclidean method where the Euclidean time $\tau$ is imaginary $\tau=i t$ and periodic of period $\beta$ which is the inverse of the temperature $\beta=T^{-1}$. The Euclidean action $I_{\text {Euc }}$ is related with the free energy $F$ by

$$
I_{\mathrm{Euc}}=\beta F=\beta(M-T \mathcal{S}-\Omega J),
$$

where $M$ is the mass, $\mathcal{S}$ the entropy and $\Omega$ is the chemical potential corresponding to the angular momentum $J$. On the other hand, in order to display the boundary term $B$ that will ensure the finiteness of the Euclidean action, we find more convenient to consider the following class of Euclidean metric

$$
d s^{2}=H^{2}(r)\left\{N^{2}(r) F(r) d \tau^{2}+\frac{d r^{2}}{F(r)}+R^{2}(r)\left(d \varphi+i N^{\varphi}(r) d \tau\right)^{2}\right\},
$$

with the assumption that the scalar field only depends on the radial coordinate, $\phi=\phi(r)$. The Euclidean time $\tau \in[0, \beta]$ and the radial coordinate $r \in\left[r_{h}, \infty\left[\right.\right.$ where $r_{h}$ is the location of the horizon and $\varphi \in[0,2 \pi[$. The reduced action principle reads

$$
I_{\mathrm{Euc}}=2 \pi \beta \int\left(N(r) \mathcal{H}(r)+N^{\varphi}(r) p(r)^{\prime}\right) d r+B,
$$

where

$$
p(r)=\frac{1}{16} \frac{H(r) R^{3}(r)\left(8-\phi(r)^{2} \kappa\right) N^{\varphi}(r)^{\prime}}{N(r) \kappa},
$$

and the Hamiltonian $\mathcal{H}$ is given by

$$
\begin{aligned}
\mathcal{H}= & \frac{8-\kappa \phi^{2}}{8 \kappa}\left[R F H^{\prime \prime}+H F R^{\prime \prime}-\frac{R F H^{\prime 2}}{H}+\frac{1}{2} F^{\prime} R^{\prime} H+H^{\prime}\left(\frac{1}{2} F^{\prime} R+R^{\prime} F\right)\right] \\
& -\frac{1}{4} H F R \phi \phi^{\prime \prime}+\frac{1}{4} H F R \phi^{\prime 2}-\frac{1}{4}\left(\frac{1}{2} F^{\prime} R+F R^{\prime}\right) H \phi \phi^{\prime}+\frac{H^{3} R(\Lambda+\kappa U(\phi))}{\kappa} \\
& -\frac{24 \kappa p^{2}}{H R^{3}\left(\kappa \phi^{2}-8\right)} .
\end{aligned}
$$

In the reduced action (3.19), $B$ is a boundary term that is fixed by requiring that the Euclidean action has an extremum, that is $\delta I_{E}=0$; this last condition in turn implies that

$$
\begin{aligned}
\delta B & =-2 \pi \beta\left[\left(\frac{8-\kappa \phi^{2}}{8 \kappa}\left\{\frac{1}{2} H^{\prime} R N+\frac{1}{2} H R^{\prime} N\right\}-\frac{1}{8} H R N \phi \phi^{\prime}\right) \delta F+\left(\frac{8-\kappa \phi^{2}}{8 \kappa} R F N\right) \delta H^{\prime}+\right. \\
& +\left(\frac{8-\kappa \phi^{2}}{8 \kappa}\left\{-2 \frac{H^{\prime} R F N}{H}-\frac{1}{2} R N F^{\prime}-R F N^{\prime}\right\}+\frac{1}{4} R F N \phi \phi^{\prime}\right) \delta H+\left(\frac{8-\kappa \phi^{2}}{8 \kappa} H F N\right) \delta R^{\prime} \\
& -\left(\frac{8-\kappa \phi^{2}}{8 \kappa} H\left\{\frac{1}{2} F^{\prime} N+F N^{\prime}\right\}\right) \delta R-\left(\frac{1}{4} H F R N \phi\right) \delta \phi^{\prime}+\left(\frac{3}{4} H F R N \phi^{\prime}+\frac{1}{8} H F^{\prime} R N \phi\right. \\
& \left.\left.+\frac{1}{4} H F R N^{\prime} \phi+\frac{1}{4} H^{\prime} F R N \phi\right) \delta \phi+N^{\varphi} \delta p\right]_{r=r_{h}}^{r=\infty},
\end{aligned}
$$


where the variation is taken between the horizon and the infinity. The temperature is fixed requiring regularity of the metric at the horizon yielding to

$$
\left.\beta\left(N(r) F^{\prime}(r)\right)\right|_{r_{h}}=4 \pi,
$$

and for the solution (3.17), one obtains

$$
T=\frac{(3-\mu) r_{h} \sqrt{1-\omega^{2}}}{4 \pi} .
$$

We do not display the field equations of the reduced action (3.19) but their full integration will reproduce the solution (3.17) with

$$
p=-\frac{\omega \mu\left(1-\lambda^{2}\right)}{\kappa\left(1-\omega^{2}\right)} r_{h}^{2} .
$$

We are now in position to compute the boundary term. Its contribution at the infinity gives

$$
\left.\delta B\right|_{\infty}=\left.\frac{2 \pi \beta \mu\left(1+\omega^{2}\right)\left(1-\lambda^{2}\right) r_{h}}{\kappa\left(1-\omega^{2}\right)} \delta r_{h} \Longrightarrow B\right|_{\infty}=\beta \frac{\pi \mu\left(1+\omega^{2}\right)\left(1-\lambda^{2}\right)}{\kappa\left(1-\omega^{2}\right)} r_{h}^{2},
$$

while at the horizon, one gets

$$
\left.\delta B\right|_{r_{h}}=-2 \pi \beta\left[\frac{4 \pi\left(1-\lambda^{2}\right) \mu}{\sqrt{1-\omega^{2}}(\mu-3) \kappa \beta} \delta r_{h}+N^{\varphi}\left(r_{h}\right) \delta p\right],
$$

and since $\Omega=N^{\varphi}(\infty)-N^{\varphi}\left(r_{h}\right)=-\omega$, we obtain

$$
\left.B\right|_{r_{h}}=\frac{8 \pi^{2}\left(1-\lambda^{2}\right) \mu}{\sqrt{1-\omega^{2}}(3-\mu) \kappa} r_{h}+2 \pi \beta \Omega p .
$$

Finally, the boundary term is given by

$$
\begin{aligned}
B & =\left.B\right|_{\infty}-\left.B\right|_{r_{h}} \\
& =\beta \frac{\pi \mu\left(1+\omega^{2}\right)\left(1-\lambda^{2}\right)}{\kappa\left(1-\omega^{2}\right)} r_{h}^{2}-\frac{8 \pi^{2}\left(1-\lambda^{2}\right) \mu}{\sqrt{1-\omega^{2}}(3-\mu) \kappa} r_{h}-2 \pi \beta \Omega p
\end{aligned}
$$

and hence the comparison between (3.23) and (3.18) permits the identification of the entropy

$$
\mathcal{S}=\frac{8 \mu \pi^{2} r_{h}\left(1-\lambda^{2}\right)}{\kappa(3-\mu) \sqrt{1-\omega^{2}}},
$$

as well as the mass, angular momentum and angular velocity that are given by

$$
M=\frac{\mu \pi\left(1+\omega^{2}\right)\left(1-\lambda^{2}\right)}{\kappa\left(1-\omega^{2}\right)} r_{h}^{2}, \quad J=-\frac{2 \pi \mu \omega\left(1-\lambda^{2}\right)}{\kappa\left(1-\omega^{2}\right)} r_{h}^{2}, \quad \Omega=-\omega .
$$

It is interesting to note that the values of the entropy, the mass and the angular momentum of the black hole solution with the super-renormalizable potential are precisely $\left(1-\lambda^{2}\right)$ time those of the black hole solution with the conformal potential [33, 34]. This is not 
surprising and can be explained as follows. On one hand, the actions being proportional with that precise factor (3.14), so that their Euclidean actions

$$
I_{\mathrm{Euc}}=\left(1-\lambda^{2}\right) \tilde{I}_{\mathrm{Euc}}
$$

On the other hand, since the temperatures and the chemical potentials are the same for both solution, $T=\tilde{T}$ and $\Omega=\tilde{\Omega}$, we have by virtue of (3.18)

$$
I_{\mathrm{Euc}}=\beta(M-T \mathcal{S}-\Omega J)=\left(1-\lambda^{2}\right) \tilde{I}_{\mathrm{Euc}}=\left(1-\lambda^{2}\right) \beta(\tilde{M}-T \tilde{\mathcal{S}}-\Omega \tilde{J}) .
$$

For completeness, we also notice that the first law of thermodynamics is satisfied

$$
d M=T d \mathcal{S}-\omega d J
$$

Once again, in order to display the role played by the gravitational soliton for computing the entropy, we construct the gravitational soliton and derive its mass. The soliton obtained from the static black hole configuration, eqs. (3.16)-(3.17) with $\omega=0$, through a double Wick rotation reads

$$
d s^{2}=-r^{2} h^{2}(r) d t^{2}+\frac{h^{2}(r) d r^{2}}{r^{2} f(r)}+r^{2} f(r) h^{2}(r) d \varphi^{2}
$$

with

$f(r)=1+(\mu-1)\left[\frac{2}{(3-\mu) r}\right]^{3}-\mu\left[\frac{2}{(3-\mu) r}\right]^{2}, \quad h(r)=\left[\lambda \sqrt{\frac{3(\mu-1)}{(3(\mu-1)-r \mu(3-\mu))}}+1\right]^{2}$.

For a Killing vector $\xi^{t}=(1,0,0)$, the surface term and the variation of the Noether potential read

$$
\begin{aligned}
\int_{0}^{1} d s \Theta^{r}= & \left(1-\lambda^{2}\right)\left[-\frac{8 \mu^{2} r}{9(3-\mu) \kappa(\mu-1)}+\frac{\mu^{2}(4 \mu-3)(\mu-3)^{4} r^{4}}{27(\mu-1)^{2}(3(\mu-1)+r \mu(\mu-3))^{2} \kappa}\right. \\
& \left.-\frac{(4 \mu-3)(\mu-3)^{2} r^{2}}{27(\mu-1)^{2} \kappa}-\frac{2 \mu}{(3-\mu)^{2} \kappa}\right], \\
\Delta K^{r t}\left(\xi^{t}\right)= & \left(1-\lambda^{2}\right)\left[\frac{8 \mu^{2} r}{9(3-\mu) \kappa(\mu-1)}-\frac{\mu^{2}(4 \mu-3)(\mu-3)^{4} r^{4}}{27(\mu-1)^{2}(3(\mu-1)+r \mu(\mu-3))^{2} \kappa}\right. \\
& \left.+\frac{(4 \mu-3)(\mu-3)^{2} r^{2}}{27(\mu-1)^{2} \kappa}\right],
\end{aligned}
$$

yielding to a mass of the soliton (1.2) given by

$$
M_{\mathrm{sol}}=-\frac{4 \pi \mu\left(1-\lambda^{2}\right)}{(3-\mu)^{2} \kappa} .
$$

Finally, it is easy to check that the formula of the gravitational entropy (3.24) is correctly reproduced by means of the Cardy-like formula (2.4) using the conserved quantities (3.25) and the mass of the gravitational soliton (3.28). 


\subsection{Lovelock AdS black holes}

In General Relativity, two of the main fundamental assumptions are the requirement of general covariance and the fact that the field equations for the metric are at most of second order. In three and four dimensions, these requirements automatically single out the gravity theory to be described by the Einstein-Hilbert action plus eventually a cosmological constant. However, for dimensions greater than four, a more general gravity theory, the so-called Lovelock theory, satisfies these standard requirements [35]. The $D$-dimensional Lovelock Lagrangian is a $D$-form constructed out of the vielbein, the spin connection and their exterior derivative and is given by

$$
\sum_{p=0}^{[D / 2]} \alpha_{p} \epsilon_{a_{1} \cdots a_{D}} R^{a_{1} a_{2}} \cdots R^{a_{2 p-1} a_{2 p}} e^{a_{2 p+1}} \cdots e^{a_{D}}
$$

where $R^{a b}=d \omega^{a b}+\omega_{c}^{a} \omega^{c b}$ is the curvature two-form, the coefficients $\alpha_{p}$ are arbitrary dimensionful coupling constants and the wedge product between differential forms is understood. We recognize the first two terms of the Lovelock Lagrangian to be proportional to the cosmological constant and to the Einstein-Hilbert piece. Being $D$-dimensional forms, the Lovelock actions are automatically invariant under the local Lorentz transformations. In addition, in odd dimension, this Lorentz gauge symmetry can be enlarged for a particular choice of the coefficients $\alpha_{p}$ to a local (A)dS or Poincaré symmetry group; the resulting Lagrangians are called Chern-Simons, see e. g. [36] for a review on Chern-Simons theory. As shown in [37], the coefficients $\alpha_{p}$ can also be chosen such that the theory has a unique AdS vacuum with a fixed value of the cosmological constant. In doing so, one yields to a series of inequivalent actions indexed by an integer $n$ with $1 \leq n \leq[(D-1) / 2]$, and given by

$$
S_{n}=-\frac{1}{2 \kappa n(D-3) !} \int \sum_{p=0}^{n} \frac{C_{p}^{n}}{(D-2 p)} \epsilon_{a_{1} \cdots a_{D}} R^{a_{1} a_{2}} \cdots R^{a_{2 p-1} a_{2 p}} e^{a_{2 p+1}} \cdots e^{a_{D}},
$$

or in tensorial form by

$$
\begin{aligned}
S_{n}= & \frac{1}{2 \kappa} \int d^{D} x \sqrt{-g}\left[R+\frac{(D-1)(D-2)}{n}+\frac{(n-1)}{2(D-3)(D-4)} L_{G B}\right. \\
& \left.+\frac{(n-1)(n-2)}{3 !(D-3)(D-4)(D-5)(D-6)} L^{(3)}+\cdots\right],
\end{aligned}
$$

where $L_{\mathrm{GB}}=R^{2}-4 R_{\alpha \beta} R^{\alpha \beta}+R_{\mu \nu \alpha \beta} R^{\mu \nu \alpha \beta}$ stands for the Gauss-Bonnet Lagrangian and $L^{(3)}$ is given by

$$
\begin{aligned}
L^{(3)}= & R^{3}-12 R R_{\mu \nu} R^{\mu \nu}+16 R_{\mu \nu} R_{\rho}^{\mu} R^{\nu \rho}+24 R_{\mu \nu} R_{\rho \sigma} R^{\mu \rho \nu \sigma}+3 R R_{\mu \nu \rho \sigma} R^{\mu \nu \rho \sigma} \\
& -24 R_{\mu \nu} R_{\rho \sigma \kappa}^{\mu} R^{\nu \rho \sigma \kappa}+4 R_{\mu \nu \rho \sigma} R^{\mu \nu \eta \zeta} R_{\eta \zeta}^{\rho \sigma}-8 R_{\mu \rho \nu \sigma} R_{\eta \zeta}^{\mu \nu} R^{\rho \eta \sigma \zeta} .
\end{aligned}
$$

Using differential forms, the field equations arising from the variation of the action (3.29) with respect to the vielbein and the spin connection read

$$
\begin{aligned}
\epsilon_{a a_{2} \cdots a_{D}} \bar{R}^{a_{2} a_{3}} \cdots \bar{R}^{a_{2 n-1} a_{2 n}} e^{a_{2 n+1}} \cdots e^{a_{D}}=0 \\
\epsilon_{a b a_{3} \cdots a_{D}} \bar{R}^{a_{3} a_{4}} \cdots \bar{R}^{a_{2 n-1} a_{2 n}} T^{2 n+1} e^{a_{2 n+2}} \cdots e^{a_{D}}=0,
\end{aligned}
$$


where $\bar{R}^{a b}=R^{a b}+e^{a} e^{b}$ and $T^{a}$ is the torsion 2 -form $T^{a}=d e^{a}+\omega_{b}^{a} e^{b}$. The spectrum of solutions of Lovelock and Chern-Simons gravity theories contain (topological) AdS black holes with interesting thermodynamical properties, see e.g. [37-42].

We now construct the spinning extension of the black hole solution of the field equations (3.30) found in [41] with planar base manifold. Its line element is given by

$$
d s^{2}=-N^{2}(r) d t^{2}+\frac{d r^{2}}{F(r)}+R^{2}(r)\left(d \varphi+N^{\varphi}(r) d t\right)^{2}+r^{2} \sum_{i=1}^{D-3} d x_{i}^{2}
$$

with

$$
\begin{array}{llrl}
N^{2}(r) & =r^{2} F(r)\left(1-\omega^{2}\right)\left(r^{2}-F(r) \omega^{2}\right)^{-1}, & N^{\varphi}(r) & =\omega\left(r^{2}-F(r)\right)\left(r^{2}-F(r) \omega^{2}\right)^{-1} \\
R^{2}(r) & =\frac{1}{\left(1-\omega^{2}\right)}\left(r^{2}-F(r) \omega^{2}\right), & F(r) & =r^{2}\left(1-\left(\frac{r_{h}}{r}\right)^{\frac{D-1}{n}}\right) .
\end{array}
$$

Skipping the details, the entropy together with the Hawking temperature read

$$
\mathcal{S}=\frac{2 \pi r_{h}^{D-2} \operatorname{Vol}\left(\Sigma_{D-2}\right)}{\kappa \sqrt{1-\omega^{2}}}, \quad T=\frac{(D-1) r_{h} \sqrt{1-\omega^{2}}}{4 \pi n}
$$

while the mass and angular momentum are given by

$$
M=\left(\frac{D-2+\omega^{2}}{2 \kappa}\right) \frac{\operatorname{Vol}\left(\Sigma_{D-2}\right) r_{h}^{D-1}}{n\left(1-\omega^{2}\right)}, \quad J=-\left(\frac{D-1}{2 \kappa}\right) \frac{\omega \operatorname{Vol}\left(\Sigma_{D-2}\right) r_{h}^{D-1}}{n\left(1-\omega^{2}\right)},
$$

and we easily check that the first law holds.

On the other hand, the corresponding soliton derived from the static black hole solution with a double analytic continuation is

$$
d s^{2}=-r^{2} d t^{2}+\frac{1}{r^{2}} \frac{d r^{2}}{f(r)}+r^{2} f(r) d \varphi^{2}+r^{2} \sum_{i=1}^{D-3} d x_{i}^{2}, \quad f(r)=1-\left[\frac{2 n}{(D-1) r}\right]^{\frac{D-1}{n}}
$$

and the mass of the soliton is computed to be

$$
M_{\mathrm{sol}}=-\frac{\operatorname{Vol}\left(\Sigma_{D-2}\right)}{\kappa(D-1)}\left(\frac{2 n}{D-1}\right)^{D-2}
$$

Finally, the gravitational expression of the entropy (3.33) matches perfectly with the Cardylike formula (2.4) with $d_{\text {eff }}=D-2$ and with the conserved quantities (3.34)-(3.35). Notice that this matching is far from trivial and deserves a certain attention for the following reason. As said in the introduction, there exists higher-dimensional extension of the Cardy formula that applied for field theory having an AdS dual, the so-called Cardy-Verlinde formula [9]. Nevertheless, as stressed in [43], the Cardy-Verlinde formula fails in general for the Lovelock AdS black holes independently of the topology of the base manifold. 


\subsection{Hyperscaling violation black hole in $D$ dimensions}

Up to now, we have only considered cases where the violating exponent is vanishing, $\theta=0$. Nevertheless, hyperscaling violation black holes are also known in the current literature, see e.g. [44-47]. It is conjectured that these solutions may have a certain interest in holographic contexts related to condensed matter physics, see e.g. [48] and [49]. For example, solutions with an hyperscaling violation exponent $\theta=D-3$ can be useful to describe a dual theory with an $\mathcal{O}\left(N^{2}\right)$ Fermi surface ( $N$ being the number of degrees of freedom).

Hyperscaling violation black holes can also be an excellent set-up to test the robustness of the Cardy-like formula (2.4) since in this case the effective spatial dimension $d_{\mathrm{eff}}$ is not longer equal to $D-2$ but will instead depend on the exponent $\theta$. A toy model in order to achieve this task is given by the Einstein-Hilbert action with a self-interacting scalar field

$$
S[g, \phi]=\int d^{D} x \sqrt{-g}\left[\frac{R}{2 \kappa}-\frac{1}{2} \nabla_{\mu} \phi \nabla^{\mu} \phi-U(\phi)\right],
$$

whose field equations read

$$
G_{\mu \nu}=\kappa\left[\nabla_{\mu} \phi \nabla_{\nu} \phi-g_{\mu \nu}\left(\frac{1}{2} \nabla_{\sigma} \phi \nabla^{\sigma} \phi+U\right)\right], \quad \square \phi=\frac{d U}{d \phi} .
$$

Indeed, for a Liouville potential of the form

$$
U(\phi)=-\frac{(D-2-\theta)(D-1-\theta)}{2 \kappa} e^{\frac{\sqrt{4 \kappa \theta} \phi}{\sqrt{(\theta-D+2)(D-2)}}},
$$

a static hyperscaling violation black hole was found in [50] with a generic value of the exponent $\theta$, and whose effective spatial dimension is $d_{\mathrm{eff}}=D-2-\theta$.

As done previously, we construct the spinning extension of the solution [50] that reads

$$
d s^{2}=\frac{1}{r^{\frac{2 \theta}{D-2}}}\left[-N^{2}(r) d t^{2}+\frac{d r^{2}}{F(r)}+R^{2}(r)\left(d \varphi+N^{\varphi}(r) d t\right)^{2}+r^{2} \sum_{i=1}^{D-3} d x_{i}^{2}\right],
$$

where

$$
\begin{aligned}
& N^{2}(r)=r^{2}\left(1-\omega^{2}\right) F(r)\left(r^{2}-F(r) \omega^{2}\right)^{-1}, \quad R^{2}(r)=\frac{1}{1-\omega^{2}}\left(r^{2}-F(r) \omega^{2}\right), \\
& N^{\varphi}(r)=\omega \frac{\left(r^{2}-F(r)\right)}{\left(r^{2}-\omega^{2} F(r)\right)}, \quad F(r)=r^{2}\left(1-\left(\frac{r_{h}}{r}\right)^{D-1-\theta}\right), \quad \phi(r)=\sqrt{\frac{\theta(\theta-D+2)}{\kappa(D-2)}} \ln (r) .
\end{aligned}
$$

Since the thermodynamics analysis is quite similar to the case of the scalar field with a super-renormalizable potential, we only sketch briefly the quantities of interest as the entropy and temperature of the solution

$$
\mathcal{S}=\frac{2 \pi \operatorname{Vol}\left(\Sigma_{D-2}\right)}{\kappa \sqrt{1-\omega^{2}}} r_{h}^{D-2-\theta}, \quad T=\frac{r_{h}(D-1-\theta) \sqrt{1-\omega^{2}}}{4 \pi},
$$

and hence the effective spatial dimensionality $d_{\mathrm{eff}}=D-2-\theta$. The mass and angular momentum of the solution are given by

$$
M=\frac{\left(\omega^{2}+D-2-\theta\right) \operatorname{Vol}\left(\Sigma_{D-2}\right)}{2 \kappa\left(1-\omega^{2}\right)} r_{h}^{D-1-\theta}, \quad J=-\frac{(D-1-\theta) \omega \operatorname{Vol}\left(\Sigma_{D-2}\right)}{2 \kappa\left(1-\omega^{2}\right)} r_{h}^{D-1-\theta} .
$$


On the other hand, the corresponding soliton is described by the following line element

$$
d s^{2}=\frac{1}{r \frac{2 \theta}{D-2}}\left[-r^{2} d t^{2}+\frac{d r^{2}}{f(r)}+f(r) d \varphi^{2}+r^{2} \sum_{i=1}^{D-3} d x_{i}^{2}\right]
$$

with the metric function and the scalar field given by

$$
f(r)=r^{2}\left\{1-\left[\frac{2}{(D-1-\theta) r}\right]^{D-1-\theta}\right\}, \quad \phi(r)=\sqrt{\frac{\theta(\theta-D+2)}{\kappa(D-2)}} \ln (r) .
$$

The mass of the soliton obtained through the quasilocal charge expression (1.2) reads

$$
M_{\mathrm{sol}}=-\frac{\operatorname{Vol}\left(\Sigma_{D-2}\right)}{2 \kappa}\left(\frac{2}{D-1-\theta}\right)^{D-1-\theta},
$$

and it is straightforward to check that the Cardy-like formula (2.4) with $d_{\text {eff }}=D-2-\theta$ fits perfectly with the gravitational entropy (3.40).

\subsection{Hyperscaling violation black hole with higher-order gravity theory}

As said before, the effective spatial dimensionality $d_{\text {eff }}$ is not always equal to $D-2-\theta$ but may have a different expression depending on the theory considered. Nevertheless, in order to corroborate the Cardy-like formula with a different value of the effective dimension, we opt for a pure quadratic gravity theory defined by the action

$$
\frac{1}{2 \kappa} \int d^{D} x \sqrt{-g}\left(\beta_{1} R^{2}+\beta_{2} R_{\mu \nu} R^{\mu \nu}\right),
$$

with field equations given by

$$
\begin{aligned}
\mathcal{G}_{\mu \nu}:= & \beta_{2} \square R_{\mu \nu}+\frac{1}{2}\left(4 \beta_{1}+\beta_{2}\right) g_{\mu \nu} \square R-\left(2 \beta_{1}+\beta_{2}\right) \nabla_{\mu} \nabla_{\nu} R+2 \beta_{2} R_{\mu \alpha \nu \beta} R^{\alpha \beta}+2 \beta_{1} R R_{\mu \nu} \\
& -\frac{1}{2}\left(\beta_{1} R^{2}+\beta_{2} R_{\alpha \beta} R^{\alpha \beta}\right) g_{\mu \nu}=0 .
\end{aligned}
$$

After a straightforward computation, one can see that the field equations admit the line element (3.39) with $\theta=D-1$ with the metric functions given by

$$
\begin{aligned}
& N^{2}(r)=r^{2}\left(1-\omega^{2}\right) F(r)\left(r^{2}-F(r) \omega^{2}\right)^{-1}, \quad R^{2}(r)=\frac{1}{1-\omega^{2}}\left(r^{2}-F(r) \omega^{2}\right), \\
& N^{\varphi}(r)=\omega \frac{\left(r^{2}-F(r)\right)}{\left(r^{2}-\omega^{2} F(r)\right)}, \quad F(r)=r^{2}\left(1-\left(\frac{r_{h}}{r}\right)^{\frac{2(D-1)}{D-2}}\right),
\end{aligned}
$$

and, where the coupling constants are tied as

$$
\beta_{1}=-\frac{(D+2) \beta_{2}}{5 D-2} .
$$

The entropy and temperature of the solution are given by

$$
\mathcal{S}=\frac{16 \pi \operatorname{Vol}\left(\Sigma_{D-2}\right)(D-1)^{2} \beta_{2}}{(5 D-2)(D-2) \kappa \sqrt{1-\omega^{2}}} r_{h}^{\frac{D}{D-2}}, \quad T=\frac{(D-1) \sqrt{1-\omega^{2}} r_{h}}{2(D-2) \pi},
$$


which imply that the effective dimension is $d_{\mathrm{eff}}=D /(D-2)$. Without giving more details, we just report the usual quantities of interest

$$
\begin{aligned}
M & =\frac{4\left(D+\omega^{2}(D-2)\right) \beta_{2}(D-1)^{2} \operatorname{Vol}\left(\Sigma_{D-2}\right)}{(D-2)^{2} \kappa(5 D-2)\left(1-\omega^{2}\right)} r_{h}^{\frac{2(D-1)}{D-2}}, \\
J & =-\frac{8(D-1)^{3} \beta_{2} \operatorname{Vol}\left(\Sigma_{D-2}\right) \omega}{(D-2)^{2} \kappa(5 D-2)\left(1-\omega^{2}\right)} r_{h}^{\frac{2(D-1)}{D-2}} \\
M_{\mathrm{sol}} & =-\frac{16 \operatorname{Vol}\left(\Sigma_{D-2}\right) \beta_{2}}{(5 D-2) \kappa}\left(\frac{D-2}{4}\right)^{\frac{D}{D-2}}\left(\frac{4}{D-1}\right)^{\frac{2}{D-2}},
\end{aligned}
$$

and again, we constat the perfect matching between the gravitational entropy and the Cardy-like formula (2.4).

\section{Testing the Cardy-like formula in the anisotropic case}

We now consider the anisotropic case which corresponds to a dynamical exponent $z \neq 1$ with our convention. In the static case, the asymptotic metric of anisotropic (Lifshitz or hyperscaling violating) black holes can be described by the following line element

$$
d s^{2}=\frac{1}{r^{\frac{2 \theta}{D-2}}}\left[-r^{2 z} d t^{2}+\frac{d r^{2}}{r^{2}}+r^{2} \sum_{i=1}^{D-2} d x_{i}^{2}\right],
$$

where now $z \neq 1$ is responsible of the anisotropy between the time and the space coordinates.

In spite of the fact that the Cardy-like formula (2.4) is also appropriate with $z \neq 1$, stationary anisotropic black hole solutions are not known in the literature. Moreover, unlike the isotropic case, the Lorentz boosts are not longer symmetries for spacetimes with $z \neq 1$, and hence the usual trick of performing a Lorentz boost to the static solution may yield to a metric with a rather obscure causal structure. These are the reasons for which we will first concentrate on static anisotropic black holes $(J=0)$ in order to test the consistency of the formulas (2.4)-(2.5). Nevertheless, in the last subsection, we will observe the effect on turning on the momentum of a static Lifshitz black hole by the usual Lorentz transformation. Making abstraction of the causal structure, we will compute the mass and angular momentum of the resulting metric and see explicitly that the Cardy-like formula with $J \neq 0$ is still consistent with the gravitational entropy.

\subsection{Lifshitz black holes with higher-order gravity theories}

We now deal with a gravity action in arbitrary dimension $D$ with quadratic-curvature corrections given by

$$
S=\frac{1}{2 \kappa} \int d^{D} x \sqrt{-g}\left(R-2 \Lambda+\beta_{1} R^{2}+\beta_{2} R_{\alpha \beta} R^{\alpha \beta}+\beta_{3} R_{\alpha \beta \mu \nu} R^{\alpha \beta \mu \nu}\right) .
$$


The corresponding field equations read

$$
\begin{aligned}
G_{\mu \nu}+\Lambda g_{\mu \nu}+\left(\beta_{2}+4 \beta_{3}\right) \square R_{\mu \nu}+ & \frac{1}{2}\left(4 \beta_{1}+\beta_{2}\right) g_{\mu \nu} \square R-\left(2 \beta_{1}+\beta_{2}+2 \beta_{3}\right) \nabla_{\mu} \nabla_{\nu} R \\
+2 \beta_{3} R_{\mu \gamma \alpha \beta} R_{\nu}^{\gamma \alpha \beta}+2 & \left(\beta_{2}+2 \beta_{3}\right) R_{\mu \alpha \nu \beta} R^{\alpha \beta}-4 \beta_{3} R_{\mu \alpha} R_{\nu}^{\alpha}+2 \beta_{1} R R_{\mu \nu} \\
& -\frac{1}{2}\left(\beta_{1} R^{2}+\beta_{2} R_{\alpha \beta} R^{\alpha \beta}+\beta_{3} R_{\alpha \beta \gamma \delta} R^{\alpha \beta \gamma \delta}\right) g_{\mu \nu}=0 .
\end{aligned}
$$

In refs. [51, 52], three families of Lifshitz black hole solutions were found. In the present case, we are only interested on the family for which the dynamical exponent $z>-(D-2)$ and described by the following line element ${ }^{2}$

$$
d s^{2}=-r^{2 z}\left[1-\left(\frac{r_{h}}{r}\right)^{\frac{z+D-2}{2}}\right] d t^{2}+\frac{d r^{2}}{r^{2}\left[1-\left(\frac{r_{h}}{r}\right)^{\frac{z+D-2}{2}}\right]}+r^{2} \sum_{i=1}^{D-2} d x_{i}^{2}
$$

The coupling constants ensuring the existence of this solution can be found in [51, 52]. For this family of solution, the entropy and temperature are

$$
\begin{aligned}
\mathcal{S} & =-\frac{2 \pi \operatorname{Vol}\left(\Sigma_{D-2}\right)}{\kappa} Q(z) r_{h}^{D-2}, \\
T & =\frac{(z+D-2)\left(r_{h}\right)^{z}}{8 \pi}
\end{aligned}
$$

with

$$
Q(z)=\frac{\left(3 z^{2}+(D-2)(D+2)\right)(D-2+3 z)(D+2-3 z)}{27 z^{4}-4(27 D-45) z^{3}-(D-2)\left[2(5 D-116) z^{2}+4\left(D^{2}-D+30\right) z+(D+2)(D-2)^{2}\right]} .
$$

Since the field equations are of higher order, we find more convenient to adopt the quasilocal formalism in order to compute the mass. This will correspond to the charge $Q$ defined in (1.2) with a Killing vector field $\xi^{t}=\partial_{t}$. In the present case, the tensor $P^{\alpha \beta \gamma \delta}$ appearing in the charge formula (1.2) is given by

$$
\begin{aligned}
P^{\alpha \beta \gamma \delta}= & \frac{1}{4 \kappa}\left(g^{\alpha \gamma} g^{\beta \delta}-g^{\alpha \delta} g^{\beta \gamma}\right)+\frac{\beta_{1}}{2 \kappa} R\left(g^{\alpha \gamma} g^{\beta \delta}-g^{\alpha \delta} g^{\beta \gamma}\right) \\
& +\frac{\beta_{2}}{4 \kappa}\left(g^{\beta \delta} R^{\alpha \gamma}-g^{\beta \gamma} R^{\alpha \delta}-g^{\alpha \delta} R^{\beta \gamma}+g^{\alpha \gamma} R^{\beta \delta}\right)+\frac{\beta_{3}}{\kappa} R^{\alpha \beta \gamma \delta} .
\end{aligned}
$$

After a tedious but straightforward computation, one obtains the expression of the mass

$$
M=-\frac{(D-2) \operatorname{Vol}\left(\Sigma_{D-2}\right)}{4 \kappa} Q(z) r_{h}^{z+D-2} .
$$

As usual, the corresponding static soliton is

$$
\begin{aligned}
& d s^{2}=-r^{2} d t^{2}+\frac{1}{r^{2}} \frac{d r^{2}}{f(r)}+r^{2 z} f(r) d \varphi^{2}+r^{2} \sum_{i=1}^{D-3} d x_{i}^{2}, \\
& f(r)=1-\left[\frac{4}{(z+D-2)}\right]^{\frac{z+D-2}{2 z}} \frac{1}{r^{\frac{z+D-2}{2}}},
\end{aligned}
$$

\footnotetext{
${ }^{2}$ The two remaining Lifshitz black hole solutions have a zero entropy.
} 
and its mass is computed to be

$$
M_{\mathrm{sol}}=z \operatorname{Vol}\left(\Sigma_{D-2}\right)\left[\frac{4}{(z+D-2)}\right]^{\frac{z+D-2}{z}} \frac{Q(z)}{4 \kappa} .
$$

It is interesting to note again that the expression of the entropy (4.5) coincides with the Cardy-like formula with $d_{\text {eff }}=D-2, J=0$ and for any value of the dynamical exponent $z$.

\subsection{Charged anisotropic black holes with two Abelian gauge fields}

In this subsection, we would like to check the charged version of the Cardy-like formula (2.5) in the anisotropic and static situation, $z \neq 1$ and $J=0$. In order to achieve this task, one considers the case of Einstein gravity with two abelian fields $A_{(i)}$ and a dilaton $\phi$ with action

$$
S=\frac{1}{2 \kappa} \int d^{D} x \sqrt{-g}\left(R-2 \Lambda-\frac{1}{2} \partial_{\mu} \phi \partial^{\mu} \phi-\frac{1}{4} \sum_{i=1}^{2} e^{\lambda_{i} \phi} \mathcal{F}_{(i)}^{2}\right),
$$

with $\mathcal{F}_{(i)}^{2}=F_{(i) \mu \nu} F_{(i)}^{\mu \nu}$ for $i=1,2$. For the following ansatz

$$
\begin{aligned}
d s^{2} & =-r^{2 z} F(r) d t^{2}+\frac{d r^{2}}{r^{2} F(r)}+r^{2} \sum_{i=1}^{D-2} d x_{i}^{2}, \\
A_{(i) \mu} d x^{\mu} & =A_{(i) t} d t, \quad \phi=\phi(r),
\end{aligned}
$$

a solution was found in [53]

$$
\begin{aligned}
F(r) & =1-m\left(\frac{r_{h}}{r}\right)^{z+D-2}+(m-1)\left(\frac{r_{h}}{r}\right)^{2(z+D-3)}, \\
A_{(1) t} & =\sqrt{\frac{2(z-1)}{z+D-2}} \mu^{-\frac{\lambda_{1}}{2}}\left(r^{z+D-2}-r_{h}^{z+D-2}\right), \\
A_{(2) t} & =-\sqrt{\frac{2(m-1)(D-2)}{z+D-4}} \mu^{-\frac{\lambda_{2}}{2}} r_{h}^{z+D-3}\left(r^{-(z+D-4)}-r_{h}^{-(z+D-4)}\right), \\
e^{\phi} & =\mu r \sqrt{2(D-2)(z-1)}, \quad \lambda_{1}=-\sqrt{\frac{2(D-2)}{z-1}}, \quad \lambda_{2}=\sqrt{\frac{2(z-1)}{D-2}},
\end{aligned}
$$

where $m, \mu$ are integration constants, and $r_{h}$ stands for the location of the horizon. Note that this presentation (4.13) is equivalent to the one considered in [53], after some redefinitions of the constants. We stick to (4.13) for latter convenience. With our notation, the Wald entropy and Hawking temperature read

$$
\begin{aligned}
\mathcal{S} & =\frac{2 \pi}{\kappa} r_{h}^{D-2} \operatorname{Vol}\left(\Sigma_{D-2}\right), \\
T & =\frac{[(z+D-4)(2-m)+2]}{4 \pi} r_{h}^{z},
\end{aligned}
$$

while the mass, electric potential and electric charge are

$$
\begin{aligned}
M & =\frac{(D-2) m}{2 \kappa} r_{h}^{z+D-2} \operatorname{Vol}\left(\Sigma_{D-2}\right), \quad \Phi_{e}=\sqrt{\frac{2(D-2)(m-1)}{z+D-4}} \mu^{-\frac{\lambda_{2}}{2}} r_{h}, \\
\mathcal{Q}_{e} & =\frac{\sqrt{2(D-2)(m-1)(z+D-4)} \mu^{\frac{\lambda_{2}}{2}}}{2 \kappa} r_{h}^{z+D-3} \operatorname{Vol}\left(\Sigma_{D-2}\right) .
\end{aligned}
$$


It remains to derive the soliton counterpart from the uncharged black hole solution which corresponds to the limit $m \rightarrow 1$. The double Wick rotation takes the following form

$$
\begin{aligned}
& d s^{2}=-r^{2} d t^{2}+\frac{d r^{2}}{r^{2} f(r)}+r^{2 z} f(r) d \varphi^{2}+r^{2} \sum_{i=1}^{D-3} d x_{i}^{2}, \\
& f(r)=1-\left(\frac{\tilde{r_{h}}}{r}\right)^{z+D-2},
\end{aligned}
$$

where we have defined

$$
\tilde{r_{h}}=\left(\frac{2}{z+D-2}\right)^{\frac{1}{z}} .
$$

Using (1.3), the variation of the Noether potential and the surface term read

$$
\Delta K^{r t}=-\frac{\tilde{r}_{h}^{z+D-2}}{\kappa}, \quad \int_{0}^{1} d s \Theta^{r}=-\frac{z-2}{2 \kappa}{\tilde{r_{h}}}^{z+D-2}
$$

and then the mass of the soliton is

$$
M_{\mathrm{sol}}=-\frac{z \operatorname{Vol}\left(\Sigma_{D-2}\right)}{2 \kappa}\left(\frac{2}{z+D-2}\right)^{\frac{z+D-2}{z}} .
$$

It is now straightforward to check that the formula (2.5) matches perfectly with the Wald entropy (4.14).

\subsection{Turning on the angular momentum}

We now turn on the angular momentum of the solution discussed in section 4.1 by operating a standard Lorentz transformation

$$
t \rightarrow \frac{1}{\sqrt{1-\omega^{2}}}(t+\omega \varphi), \quad \varphi \rightarrow \frac{1}{\sqrt{1-\omega^{2}}}(\varphi+\omega t) .
$$

The resulting metric reads

$$
d s^{2}=-N^{2}(r) d t^{2}+\frac{d r^{2}}{F(r)}+R^{2}(r)\left(d \varphi+N^{\varphi}(r) d t\right)^{2}+r^{2} \sum_{i=1}^{D-3} d x_{i}^{2}
$$

where

$$
\begin{array}{rlrl}
N^{2}(r) & =r^{2(z+1)} H(r)\left(1-\omega^{2}\right)\left[r^{2}-r^{2 z} H(r) \omega^{2}\right]^{-1}, & \\
R^{2}(r) & =\frac{1}{\left(1-\omega^{2}\right)}\left[r^{2}-r^{2 z} H(r) \omega^{2}\right], & F(r) & =r^{2} H(r), \\
N^{\varphi}(r) & =\omega\left[r^{2}-r^{2 z} H(r)\right]\left[r^{2}-r^{2 z} H(r) \omega^{2}\right]^{-1}, & H(r) & =\left[1-\left(\frac{r_{h}}{r}\right)^{\frac{z+D-2}{2}}\right] .
\end{array}
$$

As already mentioned, the resulting metric may suffer some pathology essentially due to the fact that the combination $r^{2}-r^{2 z} H(r) \omega^{2}$ is not ensured to be positive for any value 
of $r>0$ as it is the case in the isotropic situation $z=1$. Nevertheless, making abstraction of this problem, one can still compute the entropy and temperature of the solution

$$
\begin{aligned}
\mathcal{S} & =-\frac{2 \pi \operatorname{Vol}\left(\Sigma_{D-2}\right)}{\kappa \sqrt{1-\omega^{2}}} Q(z) r_{h}^{D-2}, \\
T & =\frac{(z+D-2) \sqrt{1-\omega^{2}} r_{h}^{z}}{8 \pi},
\end{aligned}
$$

where $Q(z)$ is defined in (4.7). One of the advantage of the quasilocal formalism [27, 28] is precisely to overcome the difficulty at infinity by introducing a one-parameter and by integrating in the interior region and not at infinity. Since the asymptotic form of the resulting metric (4.17)-(4.18) is not clear, the quasilocal formalism seems to be very-well appropriated to circumvent this problem. In doing so, one can compute the mass and the angular momentum

$$
\begin{gathered}
M=-\frac{\left(D-2+z \omega^{2}\right) \operatorname{Vol}\left(\Sigma_{D-2}\right)}{4 \kappa\left(1-\omega^{2}\right)} Q(z) r_{h}^{z+D-2}, \\
J=\frac{(z+D-2) \operatorname{Vol}\left(\Sigma_{D-2}\right) \omega}{4 \kappa\left(1-\omega^{2}\right)} Q(z) r_{h}^{z+D-2} .
\end{gathered}
$$

Finally, it is somehow appealing that the Cardy-like formula (2.4) with the angular momentum turning on still reproduces the correct value of the gravitational entropy.

\section{$5 \quad$ Summary and concluding remarks}

Here, we have considered rotating (an)isotropic black holes in arbitrary dimension with a planar horizon which are obtained from static configurations through a Lorentz transformation. The aim of this paper is to show that the spinning black hole entropy can be obtained from the microcanonical degeneracy of states according to a Cardy-like formula making no reference to any central charge but instead involving the mass of the ground state. The ground state is in fact identified with a gravitational bulk soliton. Hence, one of our working hypothesis in order to reproduce the semiclassical black hole entropy is the existence of a soliton. From a technical perspective, the soliton, in all the examples we have treated, is obtained from the static black hole by a double analytic continuation followed by a suitable rescaling that permits to absorb the constant of integration. This procedure is quite similar to the one that yields the AdS soliton [19]. In doing so, the resulting solitonic solution turns to be smooth, regular and devoid of any constant of integration fulfilling what the ground state is expected to be. However, there exist black hole solutions for which the double Wick rotation does not apply for different reasons. For example, this can occur for black holes for which the topology of the event horizon presents an anisotropic scaling symmetry. Such examples have been known much before the advent of Lifshitz spacetimes [54]. In this reference, two families of static black holes solutions of Einstein equations in five dimensions with a negative cosmological constant were constructed, and the horizon topologies of these solutions are modeled by the Solv 3-geometry and the Nil 3 -geometry. These geometries are two of the eight geometries of the Thurston classification. The Solv (resp. the Nil) solution is asymptotically AdS (resp. Lifshitz with $z=3 / 2$ ) 
but both solution enjoys an anisotropy along one of the coordinates of the event horizon responsible of the violation of the hyperscaling property. On one hand, a simple calculation shows that the Cardy-Verlinde formula [9] for the Solv solution does not yield the correct temperature dependence. On the other hand, while the Solv solution fits perfectly our assumptions, some complications have emerged concerning the Nil solution, in particular to construct the corresponding soliton. An interesting task will consist in understanding what would be the soliton configuration for the Nil solution or how to construct it (even numerically). One can go further extending the analysis done in this paper to the many examples of black holes with Thurston horizon topology.

Another aspect that may deserve some attention in the future has to do with the Smarr formulas. These latter are relations expressing the mass as a simple bilinear form involving the other conserved charges and the thermodynamical quantities [55]. Smarr relations can also be viewed as the integral forms of the first law of thermodynamics. For example, in all the cases studied in this paper, the solutions satisfy a Smarr relation given generically by

$$
M=\left(\frac{d_{\mathrm{eff}}}{d_{\mathrm{eff}}+z}\right) T \mathcal{S}+\Omega J .
$$

In the case of asymptotically AdS black holes, extended versions of the first law and of the Smarr formula have been obtained where the cosmological constant is considered as a thermodynamic variable, see e.g. [56, 57]. In this perspective, the mass of the AdS black hole may be understood as the enthalpy of spacetime while the cosmological constant plays the role of a pressure term in the first law. Recently, these ideas have been shown to hold also for Lifshitz black holes [58]. Since Smarr and Cardy formulas are intimately linked, it will be interesting to identify the physical implications on the Cardy-like formulas of viewing the mass as enthalpy.

Finally, we expect that the survey operating in this paper, apart from confirming the validity of the Cardy-like formulas, will be of relevance in order to clarify some issues concerning the field theory side.

\section{Acknowledgments}

We thank Eloy Ayón-Beato for useful discussions. This work has been partially supported by grant 1130423 from FONDECYT. MB is supported by "Plan de Mejoramiento Institucional" UCM1310, MINEDUC, Chile.

Open Access. This article is distributed under the terms of the Creative Commons Attribution License (CC-BY 4.0), which permits any use, distribution and reproduction in any medium, provided the original author(s) and source are credited.

\section{References}

[1] J.D. Bekenstein, Black holes and entropy, Phys. Rev. D 7 (1973) 2333 [inSPIRE].

[2] S.W. Hawking, Particle Creation by Black Holes, Commun. Math. Phys. 43 (1975) 199 [Erratum ibid. 46 (1976) 206] [INSPIRE]. 
[3] J.D. Brown and M. Henneaux, Central Charges in the Canonical Realization of Asymptotic Symmetries: An Example from Three-Dimensional Gravity,

Commun. Math. Phys. 104 (1986) 207 [InSPIRE].

[4] J.L. Cardy, Operator Content of Two-Dimensional Conformally Invariant Theories, Nucl. Phys. B 270 (1986) 186 [inSPIRE].

[5] M. Bañados, C. Teitelboim and J. Zanelli, The Black hole in three-dimensional space-time, Phys. Rev. Lett. 69 (1992) 1849 [hep-th/9204099] [INSPIRE].

[6] A. Strominger, Black hole entropy from near horizon microstates, JHEP 02 (1998) 009 [hep-th/9712251] [INSPIRE].

[7] M. Guica, T. Hartman, W. Song and A. Strominger, The Kerr/CFT Correspondence, Phys. Rev. D 80 (2009) 124008 [arXiv:0809.4266] [InSPIRE].

[8] T. Azeyanagi, G. Compere, N. Ogawa, Y. Tachikawa and S. Terashima, Higher-Derivative Corrections to the Asymptotic Virasoro Symmetry of $4 d$ Extremal Black Holes, Prog. Theor. Phys. 122 (2009) 355 [arXiv:0903.4176] [INSPIRE].

[9] E.P. Verlinde, On the holographic principle in a radiation dominated universe, hep-th/0008140 [INSPIRE].

[10] S. Detournay, L.-A. Douxchamps, G.S. Ng and C. Zwikel, Warped $A d S_{3}$ black holes in higher derivative gravity theories, JHEP 06 (2016) 014 [arXiv: 1602.09089] [INSPIRE].

[11] H.A. Gonzalez, D. Tempo and R. Troncoso, Field theories with anisotropic scaling in 2D, solitons and the microscopic entropy of asymptotically Lifshitz black holes, JHEP 11 (2011) 066 [arXiv:1107.3647] [INSPIRE].

[12] E. Shaghoulian, A Cardy formula for holographic hyperscaling-violating theories, JHEP 11 (2015) 081 [arXiv:1504.02094] [INSPIRE].

[13] M. Bravo-Gaete, S. Gomez and M. Hassaine, Towards the Cardy formula for hyperscaling violation black holes, Phys. Rev. D 91 (2015) 124038 [arXiv:1505.00702] [INSPIRE].

[14] M. Bravo-Gaete, S. Gomez and M. Hassaine, Cardy formula for charged black holes with anisotropic scaling, Phys. Rev. D 92 (2015) 124002 [arXiv:1510.04084] [INSPIRE].

[15] S. Kachru, X. Liu and M. Mulligan, Gravity duals of Lifshitz-like fixed points, Phys. Rev. D 78 (2008) 106005 [arXiv: 0808.1725] [InSPIRE].

[16] F. Correa, C. Martinez and R. Troncoso, Scalar solitons and the microscopic entropy of hairy black holes in three dimensions, JHEP 01 (2011) 034 [arXiv: 1010.1259] [INSPIRE].

[17] F. Correa, C. Martinez and R. Troncoso, Hairy Black Hole Entropy and the Role of Solitons in Three Dimensions, JHEP 02 (2012) 136 [arXiv:1112.6198] [INSPIRE].

[18] F. Correa, A. Faúndez and C. Martínez, Rotating hairy black hole and its microscopic entropy in three spacetime dimensions, Phys. Rev. D 87 (2013) 027502 [arXiv:1211.4878] [INSPIRE].

[19] G.T. Horowitz and R.C. Myers, The AdS/CFT correspondence and a new positive energy conjecture for general relativity, Phys. Rev. D 59 (1998) 026005 [hep-th/9808079] [INSPIRE].

[20] E. Ayón-Beato, M. Bravo-Gaete, F. Correa, M. Hassaïne, M.M. Juárez-Aubry and J. Oliva, First law and anisotropic Cardy formula for three-dimensional Lifshitz black holes, Phys. Rev. D 91 (2015) 064006 [arXiv:1501.01244] [INSPIRE]. 
[21] E. Shaghoulian, Black hole microstates in AdS, Phys. Rev. D 94 (2016) 104044 [arXiv: 1512.06855] [INSPIRE].

[22] L.F. Abbott and S. Deser, Stability of Gravity with a Cosmological Constant, Nucl. Phys. B 195 (1982) 76 [inSPIRE].

[23] L.F. Abbott and S. Deser, Charge Definition in Nonabelian Gauge Theories, Phys. Lett. B 116 (1982) 259 [INSPIRE].

[24] S. Deser and B. Tekin, Gravitational energy in quadratic curvature gravities, Phys. Rev. Lett. 89 (2002) 101101 [hep-th/0205318] [INSPIRE].

[25] S. Deser and B. Tekin, Energy in generic higher curvature gravity theories, Phys. Rev. D 67 (2003) 084009 [hep-th/0212292] [InSPIRE].

[26] C. Senturk, T.C. Sisman and B. Tekin, Energy and Angular Momentum in Generic F(Riemann) Theories, Phys. Rev. D 86 (2012) 124030 [arXiv:1209.2056] [INSPIRE].

[27] W. Kim, S. Kulkarni and S.-H. Yi, Quasilocal Conserved Charges in a Covariant Theory of Gravity, Phys. Rev. Lett. 111 (2013) 081101 [Erratum ibid. 112 (2014) 079902] [arXiv: 1306.2138] [INSPIRE].

[28] Y. Gim, W. Kim and S.-H. Yi, The first law of thermodynamics in Lifshitz black holes revisited, JHEP 07 (2014) 002 [arXiv: 1403.4704] [INSPIRE].

[29] J.P.S. Lemos, Cylindrical black hole in general relativity, Phys. Lett. B 353 (1995) 46 [gr-qc/9404041] [INSPIRE].

[30] A.M. Awad, Higher dimensional charged rotating solutions in (A)dS space-times, Class. Quant. Grav. 20 (2003) 2827 [hep-th/0209238] [InSPIRE].

[31] C. Martinez, C. Teitelboim and J. Zanelli, Charged rotating black hole in three space-time dimensions, Phys. Rev. D 61 (2000) 104013 [hep-th/9912259] [INSPIRE].

[32] E. Ayón-Beato, M. Hassaïne and J.A. Méndez-Zavaleta, (Super-)renormalizably dressed black holes, Phys. Rev. D 92 (2015) 024048 [arXiv: 1506.02277] [INSPIRE].

[33] C. Martinez and J. Zanelli, Conformally dressed black hole in $(2+1)$-dimensions, Phys. Rev. D 54 (1996) 3830 [gr-qc/9604021] [INSPIRE].

[34] M. Henneaux, C. Martinez, R. Troncoso and J. Zanelli, Black holes and asymptotics of $2+1$ gravity coupled to a scalar field, Phys. Rev. D 65 (2002) 104007 [hep-th/0201170] [INSPIRE].

[35] D. Lovelock, The Einstein tensor and its generalizations, J. Math. Phys. 12 (1971) 498 [INSPIRE].

[36] M. Hassaïne and J. Zanelli, Chern-Simons (super)gravity, World Scientific, Singapore (2016).

[37] J. Crisostomo, R. Troncoso and J. Zanelli, Black hole scan, Phys. Rev. D 62 (2000) 084013 [hep-th/0003271] [INSPIRE].

[38] D.G. Boulware and S. Deser, String Generated Gravity Models, Phys. Rev. Lett. 55 (1985) 2656 [InSPIRE].

[39] M. Bañados, C. Teitelboim and J. Zanelli, Dimensionally continued black holes, Phys. Rev. D 49 (1994) 975 [gr-qc/9307033] [InSPIRE].

[40] R.-G. Cai and K.-S. Soh, Topological black holes in the dimensionally continued gravity, Phys. Rev. D 59 (1999) 044013 [gr-qc/9808067] [InSPIRE]. 
[41] R. Aros, R. Troncoso and J. Zanelli, Black holes with topologically nontrivial AdS asymptotics, Phys. Rev. D 63 (2001) 084015 [hep-th/0011097] [INSPIRE].

[42] R.-G. Cai, Gauss-Bonnet black holes in AdS spaces, Phys. Rev. D 65 (2002) 084014 [hep-th/0109133] [INSPIRE].

[43] R.-G. Cai, The Cardy-Verlinde formula and AdS black holes, Phys. Rev. D 63 (2001) 124018 [hep-th/0102113] [INSPIRE].

[44] M. Cadoni and M. Serra, Hyperscaling violation for scalar black branes in arbitrary dimensions, JHEP 11 (2012) 136 [arXiv:1209.4484] [INSPIRE].

[45] M. Alishahiha, E. O Colgain and H. Yavartanoo, Charged Black Branes with Hyperscaling Violating Factor, JHEP 11 (2012) 137 [arXiv:1209.3946] [INSPIRE].

[46] P. Bueno, W. Chemissany, P. Meessen, T. Ortín and C.S. Shahbazi, Lifshitz-like Solutions with Hyperscaling Violation in Ungauged Supergravity, JHEP 01 (2013) 189 [arXiv: 1209.4047] [INSPIRE].

[47] M. Hassaïne, New black holes of vacuum Einstein equations with hyperscaling violation and Nil geometry horizons, Phys. Rev. D 91 (2015) 084054 [arXiv:1503.01716] [INSPIRE].

[48] X. Dong, S. Harrison, S. Kachru, G. Torroba and H. Wang, Aspects of holography for theories with hyperscaling violation, JHEP 06 (2012) 041 [arXiv:1201.1905] [INSPIRE].

[49] M. Alishahiha and H. Yavartanoo, On Holography with Hyperscaling Violation, JHEP 11 (2012) 034 [arXiv:1208.6197] [INSPIRE].

[50] E. Perlmutter, Hyperscaling violation from supergravity, JHEP 06 (2012) 165 [arXiv: 1205.0242] [INSPIRE].

[51] E. Ayon-Beato, A. Garbarz, G. Giribet and M. Hassaine, Lifshitz Black Hole in Three Dimensions, Phys. Rev. D 80 (2009) 104029 [arXiv:0909.1347] [InSPIRE].

[52] E. Ayon-Beato, A. Garbarz, G. Giribet and M. Hassaine, Analytic Lifshitz black holes in higher dimensions, JHEP 04 (2010) 030 [arXiv:1001.2361] [INSPIRE].

[53] J. Tarrio and S. Vandoren, Black holes and black branes in Lifshitz spacetimes, JHEP 09 (2011) 017 [arXiv: 1105.6335] [INSPIRE].

[54] C. Cadeau and E. Woolgar, New five-dimensional black holes classified by horizon geometry and a Bianchi VI brane world, Class. Quant. Grav. 18 (2001) 527 [gr-qc/0011029] [INSPIRE].

[55] L. Smarr, Mass formula for Kerr black holes, Phys. Rev. Lett. 30 (1973) 71 [Erratum ibid. 30 (1973) 521] [INSPIRE].

[56] M.M. Caldarelli, G. Cognola and D. Klemm, Thermodynamics of Kerr-Newman-AdS black holes and conformal field theories, Class. Quant. Grav. 17 (2000) 399 [hep-th/9908022] [INSPIRE].

[57] D. Kastor, S. Ray and J. Traschen, Enthalpy and the Mechanics of AdS Black Holes, Class. Quant. Grav. 26 (2009) 195011 [arXiv:0904.2765] [INSPIRE].

[58] W.G. Brenna, R.B. Mann and M. Park, Mass and Thermodynamic Volume in Lifshitz Spacetimes, Phys. Rev. D 92 (2015) 044015 [arXiv:1505.06331] [InSPIRE]. 